\title{
Dose Evaluation in Medical Staff during Interventional Radiology Procedures
}

\author{
F.A. Bacchim Neto ${ }^{1}$, A.F.F. Alves ${ }^{1}$, M. Alvarez ${ }^{1}$, M.E.D. Rosa ${ }^{1}$, C.C. Macedo de Freitas ${ }^{2}$, \\ R. Moura ${ }^{3}$, D.R. Pina ${ }^{4}$, and J.R.A. Miranda ${ }^{1}$ \\ ${ }^{1}$ Dept. of Physics and Biophysics/Biosciences Institute of Botucatu, Univ. Estadual Paulista (UNESP), Botucatu, Brazil \\ ${ }^{2}$ Dept. of Neurology and Psychiatry/Botucatu Medical School, Univ. Estadual Paulista (UNESP), Botucatu, Brazil \\ ${ }^{3}$ Dept. of Surgery and Orthopedics /Botucatu Medical School, Univ. Estadual Paulista (UNESP), Botucatu, Brazil \\ ${ }^{4}$ Dept. of Tropical Diseases and Diagnosis Imaging/Botucatu Medical School, Univ. Estadual Paulista (UNESP), Botucatu, Brazil \\ \{carlosclayton, rmoura, drpina\}@fmb.unesp.br, \{jmiranda, matheus\}@ibb.unesp.br, \\ meudelarosa@gmail.com, allan_ffa@hotmail.com, \\ bacchim_neto@aluno.ibb.unesp.br
}

\begin{abstract}
Interventional Radiology is the radiology modality that provides the highest dose values to medical staff. Recent researches show that personal dosimeters may underestimate dose values in interventional physicians, especially in extremities and eye lens. The aim of this work was to study radiation exposure levels of medical staff in different hemodynamics interventional radiology procedures. Dosimeters were positioned in different body regions of the interventional physician. Dose values were compared with annual dose limits for workers thus finding the maximum number of annual procedures that each physician could perform. As each body region of the interventionist is subject to different levels of exposure, dose distribution in each region provides a better approach to what actions are necessary to ensure the radiological protection of medical staff.
\end{abstract}

Keywords - radiation protection, dosimetry, interventional radiology.

\section{INTRODUCTION}

Interventional Radiology (IR) is the area of radiology that provides the highest doses of radiation for medical staff [1-2]. Diagnostic and therapeutic procedures in IR are performed by catheters introduced for percutaneous access and guided by fluoroscopic imaging, identifying vascular lesions and performing their treatments [3].

High doses of radiation in medical staff are due to the extreme proximity to scattered radiation source and the prolonged exposure during procedures [1-2].

Dose measurements in interventional physicians are difficult to standardize due to the great dynamism found in IR procedure. The professionals who remain closer to the patient receive the highest levels of radiation, since the patient is the main source scattered radiation [4].

Many other factors can influence the level of exposure to medical staff, as the height of the professionals, the positioning in the room, the position of the X-ray tube relative to the table and the patient, proper use of radiological protection vests and especially the total exposure time during the procedure $[2 ; 5]$.
With so many factors that can influence the exposures in occupationally exposed individuals (OEI), a detailed study of these variations to complement the radiological protection measures is crucial. Studies show that personal dosimeters may underestimate doses in OEI, especially in the extremities (hands and feet) and in the eye lens [1].

The IR provides unquestionable benefits and replaces extremely invasive and difficult recovery procedures for the patient, however, excessive exposure to radiation can cause severe damage to patients and the health care of professionals. For this reason, studies that assess the doses received under typical conditions procedures in routine sectors are essential $[4 ; 6]$. Thermoluminescent dosimeters are the most widely used radiation dosimeters due to its measurement accuracy, good proportionality response in the diagnostic radiology energy range [1-3; 7-8].

The aim of this study was to investigate the radiation exposure profiles in medical staff during different interventional procedures in Sector of Hemodynamics, Hospital das Clínicas, Faculty of Medicine of Botucatu (HC-FMB). Based on the dose profiles and the average time of each examination method, the numbers procedures each professional can perform were estimated without exceeded the annual dose limits.

\section{Materials And Methods}

The work was reviewed by the local Ethics Committe in Research and approved under the CAAE number: 16932513.5.0000.5411.

\section{A. Selection of procedures}

To choose the procedures followed in this paper two criteria were used, the total exposure time (fluoroscopy time) and the frequency they are performed. Based on these criteria, among all procedures performed in the Hemodynamics Sector of Botucatu Medical School (HC-FMB), one could select three categories: angiography, angioplasty, with or without stents fixation and angioplasty with endoprosthesis fixation (in case of stroke kind aneurysms). The groups 
were designated: diagnosis, angioplasty and aneurysms treatment, respectively.

\section{B. Dosimetry of professionals}

TLD 100 dosimeters (LIF: Mg, Ti Harshaw ${ }^{\circledR}$ ) were used for professionals dosimetry. The TLD dosimeters were calibrated in personal surface-dose equivalent Hp (0.07). For each monitored region a set of three dosimetric tablets were fixed to obtain a higher accuracy. This dosimeter has a good response for measuring the dose within the range of 10 $\mu \mathrm{Gy}$ to $1000 \mu \mathrm{Gy}[9]$.

Dosimeters were also read with a TLD Harshaw ${ }^{\circledR}$ Model 2000 using maximum reading temperature of $240^{\circ} \mathrm{C}$, with a prior thermal treatment of 10 minutes at $100^{\circ} \mathrm{C}$. In posttreatment, dosimeters were heated for 1 hour at $400^{\circ} \mathrm{C}$ and then for 2 hours at $100^{\circ} \mathrm{C}$.

In each evaluation procedures, dosimeters were placed at the primary interventional radiologist and at one assistant who remained in the room throughout the procedure. Dosimeters were fixed at the following locations: a few inches below the eye lens (on top of the surgical mask), thyroid, chest, gonads, hand (bottom of the pulse) and foot, above the radiological protection vests as lead apron and thyroid shield. Extremities monitoring occurred in only one hand and one foot, chosen according to the proximity to the irradiation source namely the most critical doses for hand and foot.

Throughout the dosimetry, each group of dosimeters $(6$ sets with 3 TLD tablets each) contained a control group (1 set with 3 TLD tablets). Each reading was subtracted from the control group dosimeters to avoid influence of background radiation.

Dosimetry techniques employed in this study did not interfered in interventional radiologist performance, nor influenced on the results of procedures for patients.

\section{Determination of the maximum number of annual procedures}

The annual dose limits are determined by the Comissão Nacional de Energia Nuclear (CNEN) in their NN 3.01 standard. Dose limits values are $20 \mathrm{mSv}$ of equivalent dose for eye lens, $500 \mathrm{mSv}$ of equivalent dose for extremities, 50 $\mathrm{mSv}$ of annual effective dose and $100 \mathrm{mSv}$ in 5 years cumulative effective dose for the whole body [10]. These dose limits were based in the ICRP Publication 60 [11].

Attenuation values for lead protection vests were based on international guidelines [6]. 2011. Based on these data were chosen as $90 \%$ attenuation of the lead vests and $60 \%$ attenuation of the protective glasses [7]. As in some regions the dose values were obtained above the lead protections, these values were corrected by attenuation of each protection.

To determine the maximum number of annual procedures, equivalent dose values in each body region were divided by the measured dose values found in dosimetry. This procedure resulted in a maximum numbers of procedures for each region of the professional. To avoid that any body region in OEI exceed the dose limits, the lowest values obtained from this calculation were used. Thus, it was found the maximum number of procedures that each OEI could perform without exceeding annual dose limits.

\section{RESULtS}

23 procedures were evaluated: 10 diagnostics, 9 angioplasty and 4 aneurysm treatment. The mean doses values for both the primary interventional radiologist (gray bars) as well as for assisting interventional radiologist (red bars), found in the diagnostic procedures, are shown in Figure 1.

Dose values found in angioplasty procedures, both the primary interventional radiologist (gray bars) as well as for assisting interventional radiologist (red bars) are shown in Figure 2.

Dose values found in the aneurysm treatment procedures, both the primary interventional radiologist (gray bars) as well as for assisting interventional radiologist (red bars) are presented in Figure 3.

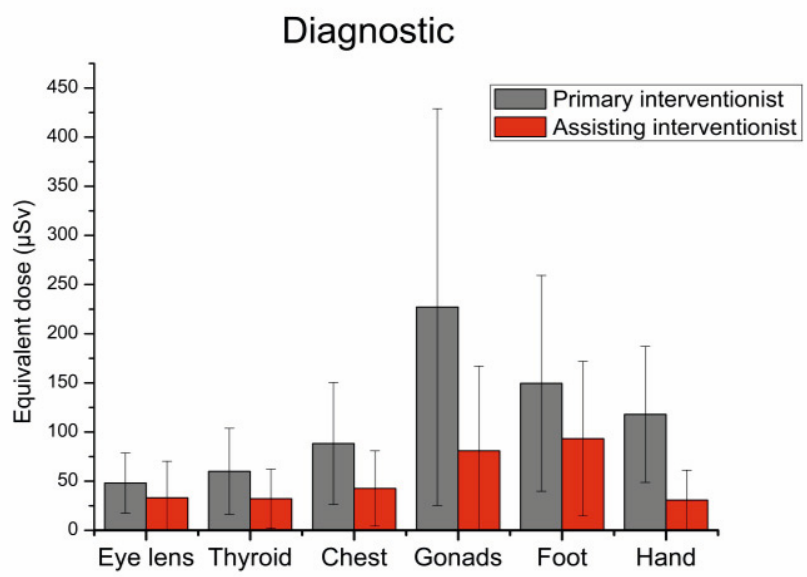

Fig. 1 Mean and standard deviation of doses per region in primary interventional radiologist (gray bars) and assisting interventional radiologist (red bars) for diagnostic procedures. 